\title{
Costos de Transacción y de Producción en Explotaciones Bovinas de la VII Región del Maule
}

\author{
LUIS SÁEZT. ${ }^{1}$, NICOLÁS GARCÍAM. ${ }^{2}$, MARIO MAINO M. ${ }^{3}$, MICHEL LEPORATI N. ${ }^{4}$
}

1 Universidad de Santiago de Chile.

2 Universidad de Santiago de Chile.

3 Universidad de Chile.

4 Ministerio de Agricultura.

\begin{abstract}
TRANSACTION COSTS AND PRODUCTION IN BEEF HOLDING OF THE MAULE REGION

The objective of this study was to determine the transaction and total cost at the beef cattle farms from Region Maule. The questionary was applied to 154 farmers with calf-beef cow system located at Curico, Talca, Parral, Longavi, Linares, Curepto, San Clemente and San Javier in April of 2008, considering aspects such us: human resources, infraestructure, farm resources, productive management, technical assistanship and trading. A conglomerated analisys was applied, with the idea to generate homogenized groups, were the farmers became distributed in 3 groups: Group 1 called beef-farmers because were totally dedicated to the cattle activity; the Group 2 dedicated bellow 50\% to beef cattle and the Group 3 having simmilar proportions of beff cattle and cereal crops. The total cost of production for kg of live weight was \$301 for Group 1, \$250 for Group 2 and \$337 for Group 3. The transactional costs represented $29 \%$ and the indirect costs only $12 \%$ of the total costs.
\end{abstract}

Key words: Transaction cost, beef production costs.

\section{RESUMEN}

El objetivo del presente trabajo fue determinar los costos de transacción y totales en las explotaciones bovinas de carne de la región del Maule. Para tal fin se realizó un estudio de campo donde se encuestó a 154 productores crianceros ubicados en las comunas de Curicó, Talca, Parral, Longaví, Linares, Curepto, San Clemente y San Javier, en Abril de 2008 considerando aspectos tales como, recursos humanos, infraestructura, recursos prediales, manejos productivos, asistencia técnica y comercialización. Se realizó un análisis de conglomerados para agrupar elementos homogéneos, donde los productores se agruparon naturalmente en tres conglomerados los cuales fueron: conglomerado 1 que se le denominó ganaderos, eran productores que sus campos estaban dedicados $100 \%$ a la ganadería; conglomerado 2 que se le denominó no ganaderos, eran productores que sus campos estaban dedicados 
en menos de un 50\% a la ganadería y conglomerado 3 que se denomino mixtos eran productores que tenían una proporción similar entre cultivos tradicionales y ganadería. Estos diferentes conglomerados tenían Costos Totales por kilo producido de \$301 para los productores del conglomerado ganadero, de \$250 para los productores del conglomerado no ganadero y de \$337 para los productores del conglomerado mixto. Referente a los Costos Totales estos están compuestos en un 59\% de los Costos Directos de Producción, los Costos de Transacción representan un 29\% y los Costos Indirectos de Producción son un $12 \%$ en la composición de los Costos Totales.

Palabras clave: Costos ganadería, costos de transacción.

\section{INTRODUCCIÓN}

El proceso de integración comercial de Chile en los mercados mundiales durante la década de los noventa se desarrolló a niveles que significaron un impacto sobre el sector pecuario. Este proceso se ha reflejado en la paulatina reducción de los aranceles generales y eliminación de medidas pararancelarias. Uno de los acuerdos más relevante desde el punto de vista de la industria bovina es el de complementación económica con el Mercado Común del Sur (MERCOSUR), el cual está compuesto por los principales competidores que tiene el sector ganadero nacional en el mercado interno. En la actualidad se está iniciando un proceso de desgravación más acelerado que debe terminar en el año 2011 con un arancel cero para los productos provenientes de este mercado. Por consiguiente, como indica Dresdner (2004), la industria bovina chilena se debe preparar para enfrentar una mayor competencia externa de precios.

Según estudio realizado por Fundación Chile (2000), el precio de la carne bovina en vara en los países del MERCOSUR, incluyendo flete y costos de internación, es de US\$2.184/TON, y el precio doméstico de la carne nacional es de US\$2.926/ TON, lo que permite prever la necesidad de adecuación del sector a este nivel de competencia que es uno de los elementos condicionantes de la dinámica futura de éste, lo cual significa no sólo poder competir con las importaciones en el país, sino además tener capacidad de competir en los mercados domésticos de otros países.

De acuerdo a los datos del censo agropecuario de 2007 (Instituto Nacional de Estadística, 2008), la producción ganadera en Chile está compuesta por 3,7 millones de cabezas de ganado concentrada preferentemente entre las regio- nes del Maule y la de los Lagos con aproximadamente el $80 \%$ de las existencias. La agricultura familiar campesina cobra relevancia estratégica ya que concentra el $42 \%$ de la existencias bovinas nacionales se Leporati et al., (2004).

La orientación productiva de las explotaciones bovinas de la agricultura familiar campesina de la región del Maule es la crianza de animales que representa $94,3 \%$, de estos productores el $84,6 \%$ se dedican a la crianza de animales para carne, los cuales se comercializan principalmente por transacciones en ferias, la segunda opción es la venta de ganado a comerciantes, las que se ven favorecidas por la posibilidad de ejecutar la venta en el mismo predio (Instituto Nacional de Estadística, 2005).

Para hacer un análisis al sector desde el punto de vista de la competitividad es necesario determinar los costos de producción y analizar, por medio de los costos de transacción, el comportamiento económico de los canales de comercialización, especialmente desde la perspectiva de las pequeñas economías campesinas, las cuales se han especializado naturalmente en la crianza, actuando como proveedores de terneros hacia el resto de la cadena (Leporati et al., 2004).

En este escenario, se desarrolló un estudio para determinar los ítems de costos más significativos que componen los costos totales anuales de las explotaciones bovinas clasificada según tipo de la agricultura familiar campesina de la región del Maule, haciendo especial énfasis en los llamados costos de transacción, entendidos estos como aquellos costos en que debe incurrir un agente, por encima de los costos de producción o de compra del bien o servicio en cuestión, para asegurar que su adquisición corresponda en mayor medida a sus necesidades o expectativas, así como la oportunidad de su entrega. 


\section{MATERIALES Y MÉTODOS}

Se realizó una investigación de campo de tipo analítica aplicada, según la clasificación propuesta por Hernández et al., (1991), que utilizo un cuestionario en terreno, con el cual se identificó, caracterizó, descompuso y relacionó las variables presentes, dándole utilidad a la recomendaciones formuladas al sector ganadero bovino.

Según los objetivos, planteados la población estudiada se conformó por las explotaciones pecuarias de la VII Región del Maule, adscritos al PABCO, en operación al momento de realizar la investigación, siendo la unidad de muestreo las explotaciones ganaderas bovinas, que resultaron ser en su totalidad usuarios de INDAP (Instituto Nacional de Desarrollo Agropecuario). Estas explotaciones se ubicaron en las comunas de Curicó, Talca, Parral, Longaví, Linares, Curepto, San Clemente y San Javier. Se determino una muestra probabilística ya que cumple con lo planteado por Hernández et al., (1991), es decir, todas las unidades de muestreo tuvieron las mismas probabilidades de ser escogidas, de manera que las mediciones en el subconjunto aportan estimados precisos del conjunto mayor, los cuales dependen del error de muestro.

\section{Tamaño de la muestra}

La determinación del tamaño de la muestra con un $95 \%$ de confiabilidad fue de 154 explotaciones bovinas, determinada mediante un muestreo estratificado según lo propuesto por Hernández et al., (1991), con el fin de que los elementos muestrales presentaran un determinado atributo, lo cual aumenta la precisión e implica el uso de diferentes tamaño de muestra para cada atributo, que para este estudio fueron las comunas anteriormente mencionadas.

El cálculo de la muestra según Hernández et al., (1991) mencionada anteriormente se detalla a continuación:

Donde:

$$
\mathrm{n}=\mathrm{Z}^{2} \mathrm{PQ} / \mathrm{d}^{2}
$$

$$
\begin{aligned}
& \mathrm{n}=\text { tamaño de la muestra } \\
& \mathrm{Z}=\text { significancia }
\end{aligned}
$$

$\mathrm{P}=$ Distribución poblacional del fenómeno la cual fue, explotaciones pecuarias crianceras de la VII Región del Maule, adscritos al PABCO.

$$
\begin{aligned}
& \mathrm{Q}=1-\mathrm{P} \\
& \mathrm{d}=\text { Coeficiente de variación }
\end{aligned}
$$

El nivel de significancia determinado en la tabla de la distribución Fisher " $z$ ", presentada por Hernández et al., (1991) es 1,96 con un grado de confianza del $95 \%$. Al no ser conocida la distribución poblacional del fenómeno se asignó el grado máximo de varianza que es de 0,5 , con el fin de reducir el error de la muestra. Por lo anteriormente mencionado y ocupando la formula $\mathrm{Q}=1-\mathrm{P}$, el valor de $\mathrm{Q}$ es de 0,5 . El error que se aceptó como permisible fue 0,05 .

De acuerdo con esto el tamaño de la muestra fue de 384 explotaciones pecuarias, debido a que esta muestra presentó un valor mayor al de la población en estudio, 256 explotaciones bovinas y que además había una restricción de recursos en cuanto a dinero y tiempo, se hizo un ajuste poblacional según Hernández et al., (1991) aplicando la siguiente ecuación:

$\mathrm{n}^{\prime \prime}=\frac{\mathrm{n}}{1+(\mathrm{n} / \mathrm{N})}$

Donde

n" = tamaño de muestra ajustada.

$\mathrm{n}=$ tamaño de muestra calculado con anterioridad.

$\mathrm{N}=$ tamaño de población

Por tanto, la muestra con un $95 \%$ de confiabilidad, fue de 154 explotaciones bovinas, lo que se distribuyó en forma probabilística por comuna, por lo cual fue necesario calcular la desviación estándar de cada elemento en un determinado estrato, lo que se obtuvo mediante el cálculo propuesto por Hernández et al. (1991) y que se presenta en la ecuación (3):

$\operatorname{sh}=\underline{\mathrm{n}}$

Donde

$$
\begin{aligned}
& \text { sh }=\text { desviación estándar de cada elemento } \\
& \text { en un determinado estrato. } \\
& \mathrm{n}=\text { tamaño de muestra definido por el estudio. } \\
& \mathrm{N}=\text { tamaño de la población }
\end{aligned}
$$

Luego para determinar el tamaño de muestra para cada comuna se utilizó la siguiente formula según (Hernández et al., 1991): 
Tabla 1. Productores encuestados por cada comuna

\begin{tabular}{lcc}
\hline Comuna & $\begin{array}{c}\text { Número } \\
\text { de productores } \\
\text { presentes }\end{array}$ & $\begin{array}{c}\text { Número } \\
\text { de productores } \\
\text { encuestados }\end{array}$ \\
\hline Curepto & 8 & 5 \\
Curicó & 26 & 16 \\
Linares & 25 & 15 \\
Parral & 92 & 55 \\
Longaví & 18 & 11 \\
San Clemente & 48 & 29 \\
Talca & 30 & 18 \\
San Javier & 9 & 5 \\
Total de productores & 256 & 154 \\
\hline
\end{tabular}

Fuente: elaboración propia.

$$
\mathrm{nh}=\mathrm{Nh}^{*} \mathrm{sh}(4)
$$

Donde

$\mathrm{nh}=$ es el tamaño de la muestra del estrato.

$\mathrm{Nh}=$ el tamaño de la población del estrato.

$\mathrm{sh}=$ es la desviación estándar de cada elemento en un determinado estrato.

La Tabla 1 presenta el número de productores encuestados en cada comuna, según la ecuación (4).

\section{Elaboración de la encuesta}

La información para el presente estudio se recopiló a través de la técnica de la encuesta a fuentes primarias, elaborada en base a información obtenida en la revisión bibliográfica y validada aplicándola a un grupo de productores, con el objetivo de determinar el tiempo de duración y la comprensión del cuestionario. La encuesta se realizó durante Abril de 2008, donde la tasa de cambio era de $\$ 446,43^{*}$.

Los aspectos analizados de los sistemas crianceros encuestados fueron recursos humanos, infraestructura, recursos prediales, estructura ganadera, manejos productivos, asistencia técnica-profesional y comercialización.

\section{Definición estructura ganadera}

Para definir la estructura ganadera de los pre- dios encuestados, se utilizó la clasificación definida en la declaración de existencia de animales del Formulario de Declaración de Existencia de Animales (Servicio Agrícola Ganadero, 2007),

\section{Tipología de ganaderos}

Para la clasificación de los productores encuestados, se utilizó el análisis de Conglomerados, el cual tiene por objeto agrupar elementos en conglomerados homogéneos en función de las similitudes entre ellos, de tal manera que la observaciones pertenecientes a un grupo sean homogéneas entre si y heterogéneas a las de otros grupos. Para ello, se utilizaron algoritmos aglomerativos que calculan las distancias entre grupos, se procedió según el método propuesto por Peña (2002).

La agrupación de los productores se realizó tomando en consideración los valores totales de las variables medidas en la encuesta (Tabla 2).

\section{Composición de los costos de transacción, costos directos e indirectos de producción}

Para cuantificar los costos de transacción y costos directos e indirectos de producción de las explotaciones pecuarias crianceras, se realizó un análisis mediante estadística descriptiva de los ítems que componen los Costos de Transacción, Costos de Transaccion por animal vendido de cada conglomerado, Costos de Transacción por Animal en cada Destino, Precio Promedio de un Animal en los diferentes Destinos, Costos Directo de Produccion por animal para cada conglomerado, Costos Indirectos de Produccion por animal para cada conglomerado y Costos Promedio Total Anual por Animal, los resultados se presentan mediante gráficos de torta y de barra, en donde en el eje horizontal se colocaron las categorías, destino 1 , destino 2 y destino 3 para los gráficos de costos de transacción promedio por animal en cada destino y precio promedio de un animal en los diferentes destinos, por otro lado para los costos promedio total anual por animal las categorías usadas fueron los conglomerados. En el eje vertical se colocó el valor monetario representado en pesos chilenos.

Para todo el análisis estadístico se ocuparon diferentes programas, en el caso de la estadística

* Disponible en www.bcentral.cl 
Tabla 2. Definición de ítems considerado para el análisis de conglomerado para

la caracterización de los sistemas crianceros

\begin{tabular}{|c|c|}
\hline Variables & Definición \\
\hline Total de Animales presente en el predio & $\begin{array}{l}\text { Consiste en los animales bovinos que estaban en el predio más los } \\
\text { animales bovinos comprados en el año, al momento de la encuesta. }\end{array}$ \\
\hline Cantidad de hectáreas dedicada a la ganadería & $\begin{array}{l}\text { Consisten en la cantidad de hectáreas empastadas más praderas } \\
\text { naturales más praderas mejoradas. }\end{array}$ \\
\hline Costo anual de mano de obra & $\begin{array}{l}\text { Considera los egresos por mano de obra dedicada a la ganadería en el } \\
\text { predio durante un año calendario. }\end{array}$ \\
\hline Costo de anual de praderas & $\begin{array}{l}\text { Considera los egresos por compras de fertilizantes que se aplican a } \\
\text { las praderas, las maquinarias ocupadas para las praderas y semillas } \\
\text { para las praderas durante un año. }\end{array}$ \\
\hline Números de animales comprados en el año & $\begin{array}{l}\text { Considera el número de animales bovinos que se adquirieron durante } \\
\text { el año. }\end{array}$ \\
\hline Costo de compra de animales en el año & $\begin{array}{l}\text { Considera el valor monetario de la variable anterior puesto en el } \\
\text { predio. }\end{array}$ \\
\hline Costo anual de alimentación & $\begin{array}{l}\text { Considera el total de egresos en: hacer silo, en la compra de fardos } \\
\text { o/y hacer fardos, compra de concentrado, compra de guano, compra } \\
\text { de sustancias anabolizantes y compra de sales minerales, en el año. }\end{array}$ \\
\hline Costo anual del manejo sanitario & $\begin{array}{l}\text { Considera el total de egreso anual por concepto de vacunas, } \\
\text { antiparasitarios y otros medicamentos. }\end{array}$ \\
\hline Destino de animales vendidos & $\begin{array}{l}\text { Considera el destino de los animales vendidos, compuesta } \\
\text { por } 3 \text { categoría que son destino } 1 \text {, venta de animales en el predio; } \\
\text { destino } 2 \text {, venta de animales a empresas que compran } \\
\text { ganado en la región, tales como Carnes Andes del sur, Carnes } \\
\text { Ñuble y Hacienda La Cantera; destino } 3 \text { venta de animales en ferias } \\
\text { ganaderas presente en la región tales como Feria Tattersal, Feria } \\
\text { Agricultores y Feria Bío-Bío. }\end{array}$ \\
\hline Número de animales vendidos & Está compuesta por la cantidad de animales que venden durante el año. \\
\hline $\begin{array}{l}\text { Dinero por concepto de ingreso } \\
\text { de animales vendidos }\end{array}$ & Está compuesta por el valor monetario de la variable anterior. \\
\hline Costos Directos de producción en el año & $\begin{array}{l}\text { Está Compuesta por la sumatoria de: manejo sanitario, } \\
\text { inseminación, compra de insumos para la mantención de praderas, } \\
\text { compra de animales, costo de alimentación, mano de obra dedicada a } \\
\text { la ganadería, asesoría técnica y mortalidad. }\end{array}$ \\
\hline Costos Indirectos de producción en el año & $\begin{array}{l}\text { Está compuesta por la sumatoria de: mantención de infraestructura, } \\
\text { pago de contribuciones, gasto en electricidad y agua de riego y pago } \\
\text { al contador. }\end{array}$ \\
\hline Costos de transacción en el año & $\begin{array}{l}\text { Está compuesta por todos los costos asociados a establecer, supervisar, } \\
\text { hacer cumplir contratos, además para este estudio se agrega el flete } \\
\text { por concepto de venta de animales. }\end{array}$ \\
\hline
\end{tabular}

Fuente: elaboración propia.

descriptiva se ocupó un software de estadística llamado "R" versión 2.4.1 donde se realizó el análisis de Conglomerado. Para la simulación de los modelos, transformación y verificación de los supuestos se utilizó el software Statistical Analysis System "S.A.S” versión 9.0.

\section{RESULTADOS Y DISCUSIÓN}

En este capítulo se presentan los principales resultados obtenidos en el estudio, en la primera parte se aborda el análisis de estadística descriptiva para caracterizar a los productores, luego se 
cuantifican los costos de transacción, directos e indirectos de producción.

\section{Caracterización de los sistemas crianceros}

El análisis de conglomerado muestra que los Productores Crianceros de la VII Región del Maule usuarias de INDAP, adscritos al PABCO se han agrupado naturalmente en tres conglomerados bien marcados, denominados "c1" que representa a los productores que poseen predios dedicados $100 \%$ a la ganadería, "c3" que representa a los productores que tienen predios dedicados mas del $50 \%$ pero menos de $100 \%$ a la ganadería y "c2" productores que poseen predios que se dedican en menos del 50\% a la ganadería.

A continuación, se analizaron las variables mencionadas en la metodología, para ver las diferencias entre los conglomerados, por medio de la dispersión y la media de cada grupo.

Existen diferencias entre los grupos, donde el conglomerado c1 tiene una mayor dispersión y cantidad de animales en comparación a los demás, ya que ellos poseen entre 62 y 211 cabezas de animales teniendo una media de 113 cabezas de animales, por otro lado el conglomerado c2 poseen entre 8 y 61 animales teniendo una media de 28 cabezas de ganado, la agrupación de productores que conforman el conglomerado c3 poseen entre 11 y 106 animales con un promedio de 39.

El grupo de productores que pertenecen al conglomerado c1 son los que destinan mayor cantidad de hectáreas a la ganadería teniendo una media de 70 hectáreas con una dispersión entre 21 y 155 hectáreas, en cambio los conglomerados c2 y c3 tienen una media de 13,5 y 23,5 hectárea dedicada a la ganadería respectivamente, con una dispersión entre 5 a 30 hectáreas para el conglomerado c2 y de 5 a 72 hectáreas para el conglomerado c 3 . Por otro lado, los primeros tres cuartiles de los productores del conglomerado c2 tienen hasta 16 hectáreas dedicadas a la ganadería, no así el conglomerado c3 que los primeros tres cuartiles tienen hasta 28 hectáreas y el conglomerado $\mathrm{c} 1$ los primeros tres cuartiles tienen 100 hectáreas dedicadas a la ganadería.

Los productores del conglomerado $\mathrm{cl}$ son los que tienen mayor Costo de Mano de Obra Anual que los demás grupos con una media de $\$ 1.909 .399$, los productores del conglomerado c3 tienen una media de $\$ 516.832$. Ya que el costo de mano de obra directa está relacionada con el tamaño de la explotación ganadera que se ve traducida en la cantidad de animales y de hectáreas dedicada a la ganadería, no así el conglomerado c2 en el que la utilización de trabajadores en el predio es más bien reducida.

No se encontraron diferencias estadísticas respecto al costo de fertilizar praderas entre los conglomerado c2 y c3, ya que el $75 \%$ de los productores que conforman estos grupos tienen un costo de $\$ 126.500$ para los productores que pertenecen al conglomerado c2 y de $\$ 150.000$ para los productores del conglomerado c3 como se muestra en la figura 4.4. Por otro lado, el costo por hectárea fertilizada para el conglomerado c1, c2 y c3 son de $\$ 8.454, \$ 6.387$ y $\$ 5.716$, respectivamente.

Las explotaciones pertenecientes al conglomerado c1 compra en promedio 9 animales anualmente, lo sigue el grupo c3 que compra en promedio 3 animales, en cambio los productores que conforman el conglomerado c2 compran en promedio 1 animal anual. Por otro lado el $75 \%$ de los productores que conforman el conglomerado c1 y c3 compran hasta 12 y 4 animales al año, respectivamente.

El costo de comprar animales es más disperso en el comglomerado c1 en donde el cuartil superior gasta por este concepto entre $\$ 2.416 .250$ y $\$ 3.365 .000$, el conglomerado c2 gasta hasta $\$ 450.000$ y el conglomerado c3 entre $\$ 325.000$ y $\$ 2.480 .000$.

Las medias de los conglomerados c1, c2, y c3 son $\$ 1.815 .000, \$ 228.047$ y $\$ 395.061$ respectivamente, lo cual indica que los productores que conforman el conglomerado c2 tienen costos de alimentación más bajo que los demás grupos, lo que se puede deber que no incurren mayormente en comprar alimento tales como guano, ensilaje o concentrado ya que los fardos que producen abastecen al ganado. No así los otros productores que se ven en la necesidad de complementar la alimentación de sus animales ya que no son capaces de abastecer los requerimientos del ganado con sus praderas.

Los productores se agrupan en función del destino donde comercializan, el 50\% de los productores del conglomerado c1 vende en el destino dos que está conformada por empresas o engorderos, las más importantes son Carnes Ñuble o Carnes Andes Del Sur; entre los engorderos a destacar está la Hacienda La Cantera y engor- 
deros de la zona, el 25\% vende en el destino 3 que está conformada por las ferias ganaderas, las cuales pueden ser la Feria de Agricultores de cada zona, la Feria Tattersal o la Feria Bío-Bío. El grupo 2 vende en los diferentes lugares ya sea ferias ganaderas (destino 3), particulares (destino 1) o a empresas (destino 2), por otro lado los productores que conforman el conglomerado c3 venden preferentemente a las ferias ganaderas.

El conglomerado c1 tiene un promedio de 29 animales vendidos y coincide con el que posee la mayor cantidad de animales. Los conglomerados c2 y c3 no presentan diferencias estadísticas respecto a la cantidad de animales vendida, ya que estos poseen una media de 7 animales y el $75 \%$ de los productores que componen los conglomerados c2 y c3 venden menos de 9 animales.

El comportamiento de los conglomerados c2 y c3 no presentan diferencia estadística respecto al dinero recibido por concepto de ingreso de animales vendidos, ya que el cuartil superior de ambos conglomerados es similar, los productores que se encuentran en el conglomerado $\mathrm{c} 1$ poseen una mayor dispersión la que se encuentra entre $\$ 1.401 .000$ y $\$ 10.605 .000$.

Los costos directos de producción muestran conglomerados bien determinados, siendo el conglomerado c1 el que posee los costos más elevado teniendo una media $\$ 6.100 .00$ con una concentración en las observaciones esto quiere decir que los productores que conforman este grupo tienen costos directos parecidos. El conglomerado $\mathrm{c} 2$ es el que posee los costos directos mas bajos que los demás grupos con un promedio de $\$ 1.900 .000$ y los productores están concentrados, lo cual indica que tienen costos parecidos entre los productores que conforman este conglomerado. El conglomerado c3 presenta una media de \$1.932.266 y se muestra menos concentrado que los conglomerados c1 y c2.

\section{Cuantificar Costos de Transacción, Directos, Indirectos de producción y Costos Totales}

\section{Determinación Costos de Transacción}

Los costos de transacción en promedio son de $\$ 151.654$ y están compuestos por el costo de conocer los precios, costo de visita de predio, costos por ofrecer animales, costo de negociar los precios, tiempo destinado de compra y/o venta, costo de transporte de animales y descuentos realizados por venta y/o compra. Dentro de los ítems que componen los costos de transacción los más relevantes son los costos de conocer los precios con una incidencia de $31 \%$ promedio, descuento realizados representa en promedio un $30 \%$ en la composición de los costos de transacción y el costo de flete representa un $25 \%$. Estos ítems son los que tienen mayor peso en la composición de los costos de transacción.

El análisis por conglomerado de los Costos de Transacción por animal vendido es $\$ 16.120$ para los productores pertenecientes para el conglomerado c1, para el conglomerado c2 es $\$ 20.323$ y para el conglomerado c3 es $\$ 21.120$. la diferencia existente entre el conglomerado $\mathrm{c} 1$ y los otros dos conglomerados radica principalmente en dos ítems que son el costo de conocer los precios y el costos de flete, ya que estos en promedio son de $\$ 1.500$ por animal mas bajo que él de los otros conglomerados, puesto que el conglomerado $\mathrm{c} 1$ vende mayormente al destino 2, lo cual trae consigo mayor acceso a la información a un bajo costo y por otro lado son capaces de llenar un camión entero cuando realizan la venta lo que genera economía de escala en el traslado de ganado.

\section{Costos de Transacción y precio de venta por destino}

Los costos de transacción por animal, que al momento de la venta pesa entre 250 y 350 kilos, en el cual el destino 3, que representa a las ferias ganaderas de la región, está asociado a un valor más alto llegando a ser de $\$ 27.900$ por animal vendido en feria, producto que cobran una comisión de un $6 \%$ por realizar la venta y un pronto pago, adelanto en efectivo del dinero de la venta, de un $1,5 \%$; por otro lado el flete que tienen que pagar los productores por el traslado de los animales es el más alto ya que los predios de estos se encuentran alejados de las ferias. Además, hay que considerar que los animales llegan antes de las 8 de la mañana y son pesados al medio día para la venta, por ende existe una pérdida de peso que no se paga al productor, lo cual trae consigo una disminución en el ingreso recibido por cada animal vendido. El destino 2, que es la venta de animales a empresas presente en la zona, tiene un costo de transacción asociado 
de $\$ 14.800$, ya que estas empresas aplican un destare $^{* *}$ de un $1 \%$ por cada 100 kilos, además el costo de traslado de sus animales es menor que el destino 3 ya que el agricultor lleva sus animales al predio de otro productor que hace de acopiador para que estas empresas pasen a buscar a los animales, en consecuencia, el costo de flete es bajo puesto que generalmente los productores se encuentran relativamente cerca del agricultor que hace de acopiador. Por otro lado, el destino 1 que representa la venta de animales a particulares, presenta costos de transacción menores que los demás destinos con $\$ 10.712$, ya que el costo de traslado de animales es mínimo o simplemente no se presenta, por cuanto la venta se realiza en el mismo predio de origen del animal, sin embargo, esta forma de comercialización debe tener manejo de información para poder fijar el precio de venta del animal.

Respecto del precio promedio de un animal en los diferentes destinos, se puede apreciar que la venta de animales a particulares (destino 1 ), es el que alcanza el mayor precio promedio de un animal que pesa entre 250 y 350 kilos llegando a ser de $\$ 187.700$, sin embargo, este destino es mas bien ocasional y no hay grandes transacciones en número de animales. Por otro lado, las ferias (destino 3) presenta un precio promedio de $\$ 181.300$; este destino es donde se comercializa la mayor cantidad de animales; la venta de animales a empresas compradoras de animales (destino 2) tiene un precio promedio de animal de $\$ 167.500$.

Si se comparan los costos de transacción de un animal promedio en cada destino con el precio promedio de cada animal en el lugar de venta se tiene que los productores que venden en el destino 1 perciben ingresos promedio de $\$ 177.000$, los que venden en el destino 2 reciben ingresos $\$ 152.700$ y los que venden en el destino 3 reciben $\$ 153.300$, la diferencia que hay en vender animales a las empresas versus vender en las feria es de $\$ 500$ por animal vendido, siendo esta última el destino de venta preferido de los productores ya que en las ferias están disponibles todo el año y se puede vender cualquier categoría de animal ya sea vaca, vaquilla, novillos, ternero, terneras, toros y bueyes, no así en las empresas que tienen periodos de compra determinados y requisitos específicos de tipos de animales; sin embargo, las empresas tienen precios diferenciales para animales que tengan ciertas características, lo cual indica que eventualmente los precios promedio de venta de animales podrían subir si cumplen esas características.

\section{Determinación Costos Directos}

Los ítems que componen los Costos Directos de Producción son: Costo de Asistencia Técnica, Costos de Fertilizar praderas, Costo de Mano de obra, Costo de Compra de animales, Costo de Alimentación, Costo de Manejo Sanitario, Costo de Manejo Reproductivo y Costo de Mortalidad, de los cuales los mas relevante para el conglomerado c1 son, la compra de animales ya que representa el 35\% de los costos de producción, en segundo lugar de importancia está el costo de Mano de obra que equivale al $28 \%$ del costo de producción y en tercer lugar está el costo de alimentación que es $23 \%$ de la composición de los costos de producción para los productores que conforman al conglomerado $\mathrm{c} 1$.

Los Costos Directos de Producción para los productores del conglomerado c3 se descompone de la siguiente manera según nivel de importancia: el costo por la compra de animales representa un $30 \%$ de los costos directos, el costos de mano de obra es $27 \%$ de la composición de los costos directos y el costos de alimentación representa un 23\% de los costos.

Para los productores que conforman el conglomerado c 2 el costo de alimentación representa un $44 \%$ de los costos de producción, lo sigue el costo de fertilizar pradera que tiene una importancia de un $17 \%$ en la composición de los costos de producción. Este conglomerado presenta diferencia en los ítems que son relevantes para los otros dos conglomerados, debido que el conglomerado c2 no está dedicado mayormente a la producción ganadera por eso el costo de compra de animales representa un $9 \%$ comparado con el $30 \%$ que representa para los otros conglomerados, el costo de mano de obra que los productores del conglomerado c2 dedican a la ganadería es mas bien reducido.

** destare: rebaja de peso del animal, producto del stress del viaje, no alimentación y deshidratación, ya que está a la espera de ser vendido. 


\section{Costos Indirectos de producción}

El conglomerado c1 es el que tiene los Costos Indirectos de Producción más elevado, ya que los productores que conforman este conglomerado son los mas grande en producción ganadera comparado con los otros productores de los diferentes conglomerado, teniendo un costos indirecto de producción de $\$ 12.139$ por animal, lo sigue el conglomerado c3 que tiene un costo indirecto de producción por animal de $\$ 10.401$ y el conglomerado c2 con $\$ 7.815$ por animal. El ítem más relevante que compone los costos indirectos de producción para los tres conglomerados es el costo del agua de riego.

\section{Costos Totales}

A continuación, se presentan los costos totales, en función de los conglomerados. Al respecto el costo total promedio por animal indica que los productores del conglomerado c1 son los que poseen mayores costos para producir un animal llegando a ser de $\$ 108.600$, los productores del conglomerado c3 poseen costos de producción por animal de $\$ 99.473$ y el conglomerado c2 tienen costos de producción por animal de $\$ 49.593$. Además se puede apreciar que los Costos de Transacción para el conglomerado c1 son mayores que los Costos Indirectos de producción en un $25,1 \%$, para los productores del conglomerado c2 los Costos de Transacción son mayores que los Costos Indirectos de Producción en un 61,5\% y para los productores del conglomerado c3 los Costos de Transacción son mayores que los Costos Indirectos de Producción en un 50,7\%.

A modo de conclusión del presente trabajo podemos decir.

a) Es posible agrupar a los productores en diferentes conglomerados, los que poseen explotaciones que se dedican completamente a la ganadería; los que poseen explotaciones que se dedican en mayor medida a la ganadería pero combinan sus actividades con cultivos tradicionales y los que desarrollan la ganadería como complemento de otras actividades principales.

b) Los productores que se dedican completamente a la ganadería tienen mayores posibilidades de vender en las empresas que compran ganado en la región como son Carnes Ñuble, Hacienda La Cantera y Carnes Andes Del Sur, ya que cuentan con ciclos productivos regulares y cumplen con las exigencias productivas de dichas empresas.

c) Los destinos no perciben un elemento diferenciador de los terneros que provienen de cada conglomerado, por otro lado, estos ofrecen diferentes precios siendo la venta a particulares el que alcanza un mayor precio por animal, seguido de las ferias ganaderas y la venta a empresas que compran ganado en la región que son las que pagan el precio más bajo por animal.

d) La venta de animales a productores es el destino que presenta menores costos de transacción con $\$ 10.712$, el destino de venta de animales a empresas presente en la zona presenta costos de transacción de $\$ 14.800$ y la venta de animales en ferias ganaderas presenta un el costo de transacción más alto con $\$ 27.900$.

e) Al descontar los costos de transacción al valor pagado por animal en cada destino, el ingreso por venta en la feria ganadera es superior en $\$ 500$ por animal, comparado con el ingreso por venta a empresas que compran ganado en la región.

f) Los Costos de Transacción de las explotaciones crianceras de la región del Maule en promedio son mayores en un $15,5 \%$ que los Costos Indirectos de Producción.

g) Es posible predecir el comportamiento de los costos de transacción, costos indirectos de producción anuales y costos directos de producción, generando modelos predictivos y explicativos, con una variabilidad de un $84,7 \%, 88,1 \%$ y de $83,7 \%$ respectivamente.

h) Para el modelo de Costos de Transacción las variables más influyentes son, el número de novillos vendidos, numero de terneros vendidos, numero de vacas vendidas, número de vaquillas vendidas, el costo de conocer el precio y el destino del animal.

i) Los costos directos de producción son lo que tienen mayor incidencia en los costos totales para producir un animal, seguido de costos de transacción.

j) Los ítems que explican mayormente la variabilidad de los costos directos de producción son, el total de animales, costos de mano de obra, el total de animales comprados y el cos- 
to de alimentación.

k) Las variables más significativas para los Costos Indirectos de Producción son, total de animales que transitan por el predio, numero de hectáreas propias y gastos en agua de riego.

1) Las variables que mas influyen en los Costos Totales de Producción son, el número de novillos vendidos, numero de terneros vendidos, número de vacas vendidas, numero de vaquillas vendidas, el costo de conocer el precio, el destino del animal, total de animales que transitan por el predio, numero de hectáreas propias, gastos en agua de riego, el total de animales, costos de mano de obra, el total de animales comprados y el costo de alimentación.

\section{REFERENCIAS}

1.- DAZA J. (2006). Estadística Aplicada. Ed $1^{\circ}$. Lima. Editorial Megabyte.

2.- DEPETRIS E, LÓPEZ R, QUAGLIANI A. (2003). Cambios Institucionales y Costos de Transacción en el Comercio de Granos. Estudio. Facultad de Ciencias Agrarias. Universidad Nacional de Rosario. Argentina.

3.- DRESDNER J. (2004). La Industria Bovina en Chile: Enfrentando las Desventajas Comparativas. Ciencia e Investigación Agraria 31 (1): 50-63.

4.- ESCOBAL J. (2000). Costos de Transacción en la Agricultura Peruana: Una Primera Aproximación a su Medición e Impacto. Lima, Perú. Documento de trabajo $\mathrm{N}^{\circ}$ 30. p 9-13.

5.- ESLAVA R. (2003). Sistema de Acumulación de Costos del Sector Ganadero Bovino del Municipio Alberto Adriani del Estado Meridia. Tesis Magister Scientiae en Administración. Venezuela. Universidad de los Andes, Facultad de Ciencias Económicas y Sociales. P 12-31.

6.- FIGUERAS A. (2001). Ronald Coase, a cuarenta años de un artículo. Actualidad Económica 11(51): 1-7.

7.- Fundación Chile (2000). El Mercado Mundial de Carnes Bovina y Ovina desde la perspectiva de Chile. Primera
Edición., Fundación Chile, Área Agroindustria. Chile.

8.- GONZÁLEZ M, PAGLIETTINI L. (2002). Los Costos Agrarios y Sus Aplicaciones. 1 ed. Argentina. Editorial Facultad de Agronomía, Universidad de Buenos Aires. P 3-23.

9.- HERNÁNDEZ RS, FERNÁNDEZ CC, BAPTISTA PL. (1991). Metodología de la Investigación. Segunda Edición., Editorial McGraw-Hill Interamericana. México.

10.- HORNGREN C, GARY L, WILLIAM O. (2006). Contabilidad Administrativa. Traducción Brito, J. México. Editorial Pearson Education. I.

11.- Instituto Nacional de Estadística, Cl. 2008. Base de datos CENSO agropecuario (en línea). Santiago, Cl. Consultado el 5 de sep. 2007. Disponible en http:// www.ine.cl.

12.- Instituto Nacional de Estadística, cl. 2005. Ganadería Bovina en la Agricultura Familiar Campesina.

13.- LEPORATI M. (2004), Formulación y Evaluación del Proyecto de Desarrollo de Encadenamiento Productivo Entre Pequeños Productores Pecuarios Beneficiarios de INDAP y la Cadena de la Carne Bovina, Departamento de Ingeniería Industrial.

14.- LEPORATI M, VILLALOBOS P, SÁEZ L,VILLAR, M. (2004). Efecto de los costos de transacción asociados a exigencias de calidad, sobre la rentabilidad de sistemas pecuarios de pequeños agricultores. I Congreso Regional de Economista Agrario. Noviembre. Mar del Plata. Argentina.

15.- MATTHEWS L. (1984). Estimación de Costos de Producción. Ed 1, México. Editorial Mc Graw Hill.

16.- NAVARRO J. (2005). Tópicos de Producción Bovina. Ed $1^{\circ}$, Fundación Chile, Área Agroindustria.

17.- PEÑA D. (2002). Análisis de Datos Multivariantes. Ed $1^{\circ}$, Madrid. Editorial Mc Graw Hill.

18.- RHESE C. (1970). Manual de Costos de Producción Ganaderos. Ed $1^{\circ}$. Buenos Aires. Editorial Selcon. P 22-75.

19.- Servicio Agrícola Ganadero. 2007. Formulario de Declaración de Existencia de Animales. Consultado 5 de sep de 2007. Disponible en. http://www.sag.gob.cl/.

20.- WEISBERG S. (2005). Applied Linear Regression. Ed $3^{\circ}$. Minneapolis, Minnesota. Editorial Wiley-Interscience.

21.- WILLIAMSON O. (1989). Las Instituciones Económicas del Capitalismo. Traducción Suárez, E. México. Editorial The Free Press. P. 26-51. 